\title{
150 cancer surgeries under hypnosis: a valuable alternative to preserve the ecology of the patients
}

\author{
A. Marcou ${ }^{1}$, J. Muret ${ }^{1}$, S. Alran ${ }^{2}$, G. Dhonneur ${ }^{1}$, P. Goater ${ }^{1}$ \\ ${ }^{1}$ Department of Anesthesiology and Intensive Care, ${ }^{2}$ Department of Breast Surgery, Institut Curie, Paris, France
}

\section{BACKGROUND AND GOAL OF STUDY}

Hypnosedation combines hypnosis + conscious sedation + locoregional anesthesia
A valuable alternative to general anesthesia decreasing medication
reducing adverse effects
fastening postoperative rehabilitation

It minimizes effects of anesthesia on vital functions while preserving the patient's well being

Can this innovative technique be currently used in a cancer center?

\section{MATERIALS AND METHODS}

Retrospective study on 150 patients operated on under hypnosis between 2011 and 2017

No experience in hypnosis was required

\section{Procedure}

- hypnosis

- a target-controlled infusion of remifentanil

\pm a local/loregional anesthesia depending to the surgery: a tumescent technique or a paravertebral block (breast surgeries), a cervical block (gynecological surgeries)

- no premedication or hypnotic drugs

- usual safety conditions and monitoring

- usual preventive antiemetics and painkillers

\section{RESULTS AND DISCUSSION}

Types of surgeries: breast, gynecological surgeries

The mean surgical duration was 60 min (30 to 160 $\min$ ).

$\checkmark$ The mean length of stay in the recovery room was $\mathbf{3 5} \mathbf{~ m i n}$.

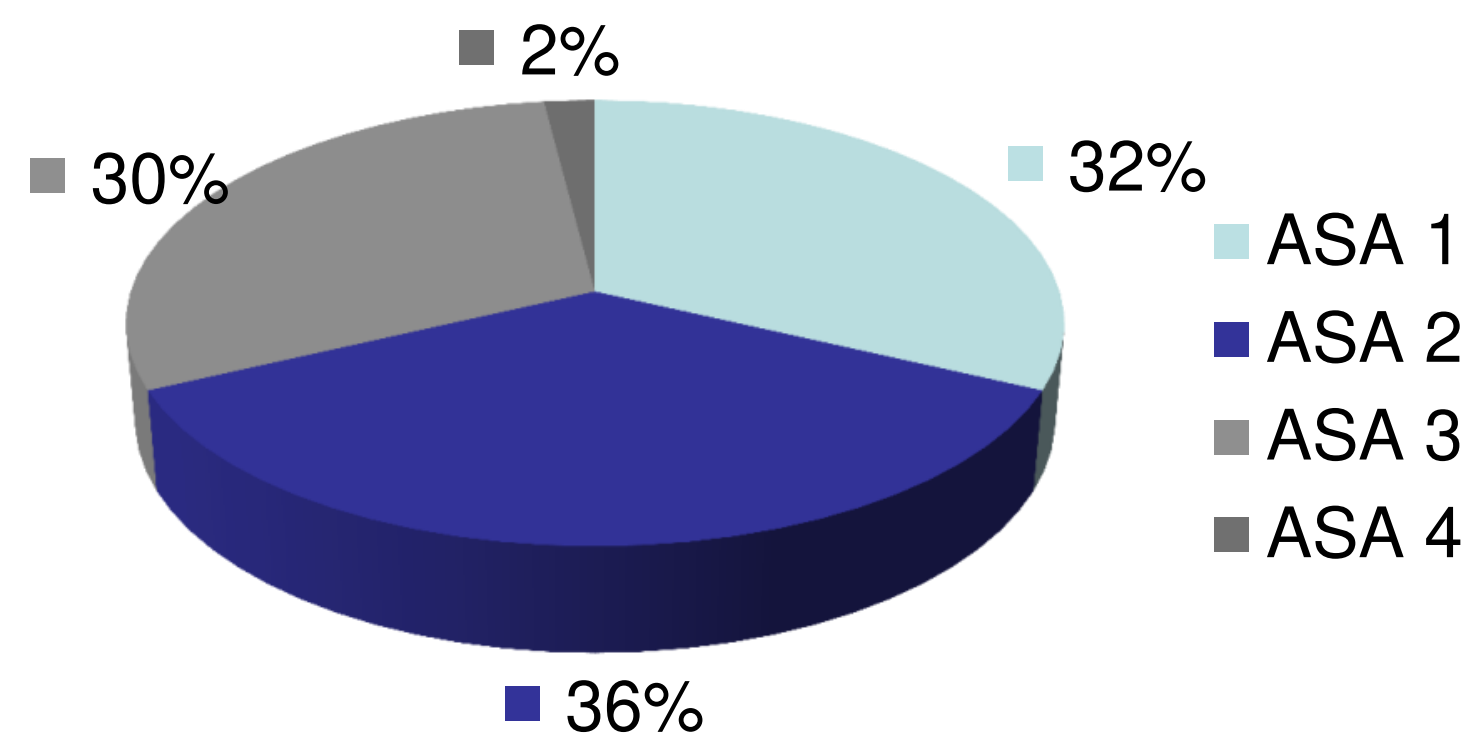

The average age was 60,5 [18-100]

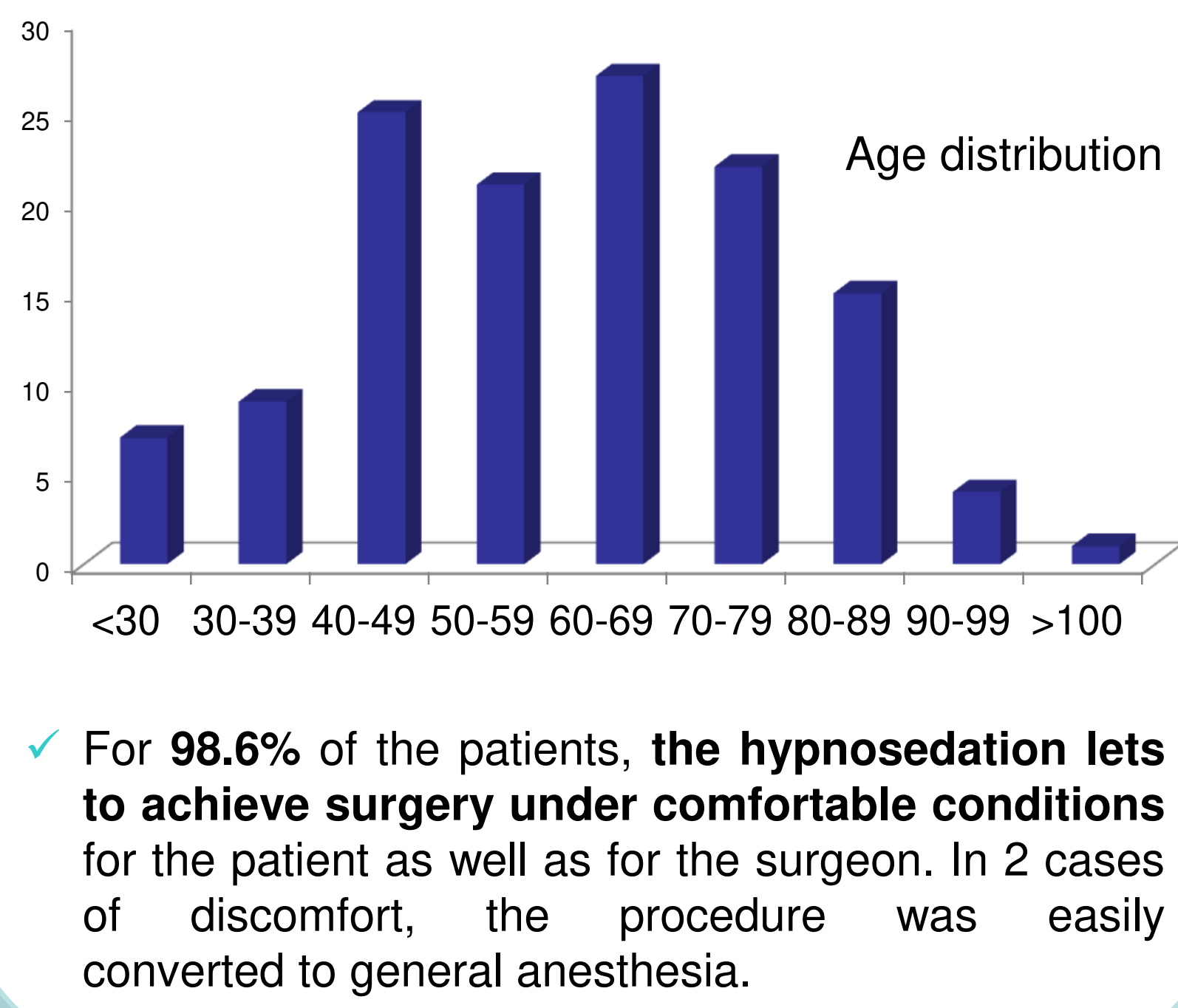

Hypnosedation can be proposed as a useful alternative to a general anesthesia in various types of surgeries, including major breast surgeries.

By minimizing effect of anesthesia, this technique contributes to preserve the ecology of the patient and is particularly valuable for vulnerable patients. 